\title{
NOTES ON CHINESE BAUHINIA WITH DESCRIPTION OF THREE NEW SPECIES
}

\author{
Luetta Chen
}

In Determining recent Chinese collections sent to the Arnold Arboretum for identification, I have found three new species of Bauhinia and four species which represent new records for certain provinces. The material on which this paper is based is deposited in the herbarium of the Arnold Arboretum.

Bauhinia aurea Lévl. Bull. Soc. Bot. France 7: 368. 1907.— L. Chen, ${ }^{1}$ Lingnan Sci. Jour. 18: . . 1939.

C.W.Wang 79234 is a new record for Yunnan.

Bauhinia caterviflora L. Chen, Jour. Arnold Arb. 19: 129. 1938. L. Chen, Lingnan Sci. Jour. 18: . . 1939.

$Y u$-shih Liu $1351 A$ is a new record for Szechuan.

\section{Bauhinia claviflora, sp. nov.}

Frutex, ut videtur, erectus; ramulis teretibus, brunneis, pubescentibus; foliis ovatis, subcoriaceis, $9.5-11 \mathrm{~cm}$. longis, $8.5-10 \mathrm{~cm}$. latis, basi cordatis, obtusis, apice emarginatis, supra glabris, ad nervos subtus pubescentibus, 13-nerviis, nervis utrinque prominentibus; venulis subreticulatis; petiolis puberulis, $2.5-3 \mathrm{~cm}$. longis; inflorescentiis racemosis, pilosis, paucifloris; bracteis binis, caducis; pedicellis crassis, $5.5 \mathrm{~cm}$. longis, pubescentia rubiginosa conferta indutis; calycis tubo $3 \mathrm{~cm}$. longo, pubescente; floribus magnis albis, ad basim rubris; calyce lobato, lobis lanceolatis, $3 \mathrm{~cm}$. longis, $0.3 \mathrm{~cm}$. latis, crassis, tomentosis, longe acuminatis; petalis 5 , extus conferte adpresseque sericeo-tomentosis, intus sparse pubescentibus, subcoriaceis, ungue $2 \mathrm{~cm}$. longa, limbo rugoso, 3 $\mathrm{cm}$. longo, $2.2 \mathrm{~cm}$. lato, emarginato; nervis supra prominentibus; staminibus fertilibus 3 , filamento crasso, glabro, circa $3.5 \mathrm{~cm}$. longo, $0.15 \mathrm{~cm}$. lato, anthera 2-loba, parcissime pubescente, $1.2 \mathrm{~cm}$. longa; filamentis sterilibus 2 , gracilibus, glabris, circa $1.5 \mathrm{~cm}$. longis; ovario stipitato, pube-

${ }^{1}$ For full literature citations, synonymy and distribution see my paper "Bauhinia in China" in Lingnan Sci. Jour. 18: ... 1939. EDitor's nOTE: The Arnold Arboretum has not received to date this publication. 
scentia densa rubescente induto; stylo crasso, ca. $2 \mathrm{~cm}$. longo, stigmate lato, ca. $0.4 \mathrm{~cm}$. diametro; legumine ignoto.

Yunnan: Meng-soong, Dah-meng-lung, Che-li Hsien, C. W. Wang 78485 , Sept. 1936, in border of woods, alt. $1800 \mathrm{~m}$.

This species is very distinct being strongly characterized by its flowers having five unusually long calyx lobes. The petals are subcoriaceous and silky pubescent on the outside.

Bauhinia corymbosa Roxb. Hort. Beng. 31. 1914, nom. nud.; Fl. Ind. 2: 329. 1832.— L. Chen, Lingnan Sci. Jour. 18: . . 1939.

S. P. Ko 55323 is a new record for Kwangsi.

\section{Bauhinia dioscoreifolia, sp. nov.}

Frutex scandens, $3 \mathrm{~m}$. altus; ramis teretibus, breviter atque sparse pubescentibus; foliis ovatis, subchartaceis, $9-13.5 \mathrm{~cm}$. longis, $7-10.5 \mathrm{~cm}$. latis, utrinque glabris, ad apicem breviter obtuseque acutatis, basi truncata vel subcordata, 7-nerviis; nervis conspicuis, utrinque elevatis; venulis utrinque minute reticulatis; petiolis $2.5-5 \mathrm{~cm}$. longis, glabris infima et superiore partibus parce pubescentibus exceptis. Inflorescentiis atque floribus haud visis; legumine ligneo, stipitato, oblongo, glaberrimo, 14-16 cm. longo, $3.5-4 \mathrm{~cm}$. lato, subcorrugato, apice breviter acuminato, basi attenuato; seminibus 5-6.

Hainan: Kumyun, S. K. Lau 27552, Sept. 7, 1936, in dense woods; fruit green.

Bauhinia erythropoda Hay. and B. dioscoreifolia L. Chen are somewhat alike in certain aspects but the 9-nerved leaves of the former are cordate and ferruginous pubescent on the nerves on the lower surface. The flowers of $B$. dioscoreifolia are, as yet, unknown, but when these are available, the two species may prove to be not at all closely related.

\section{Bauhinia megacarpa, sp. nov.}

Frutex scandens, ramulis tomentulosis; foliis late orbiculatis, subcoriaceis, 14-16 cm. longis, 14-17 cm. latis, brunneis, supra glabris, subtus grosse tomentosis, apice bilobis, lobis $1 / 3$ connatis, abrupte rotundatis, ad basim late cordatis, margine repandulis, 11-13-nerviis, nervis subtus elevatis, venulis utrinque reticulatis; petiolis pubescentibus, demum glabrescentibus, 5-6 cm. longis; inflorescentiis haud visis; legumine ligneo, stipitato, perglabro, nitido, nigro, oblongo, ca. $22 \mathrm{~cm}$. longo, $6 \mathrm{~cm}$. lato apice breviter acuminato, base attenuato; seminibus 8 obovatis compressis, ' 2 ' $\mathrm{cm}$. longis, $1.5 \mathrm{~cm}$. latis, margine laevibus, in medio corrugatis. 
HaInan: Nam Shan Leng, Yai-hsien District, S. K. Lau 5848, April 7-30, 1935, rare, on dry steep slopes in sandy soil.

This species somewhat resembles Bauhinia aurea Lévl. in leaf characters but the legume of $B$. megacarpa is larger, about $22 \mathrm{~cm}$. long and $6 \mathrm{~cm}$. broad, and wholly glabrous.

Bauhinia subrhombicarpa Merr. Lingnan Sci. Jour. 14: 9. 1935.-

L. Chen, Lingnan Sci. Jour. 18: . . 1939.

S. K. Lau 28618 is a new record for Kwangsi.

Arnold Arboretum,

HARVARD UNIVERSITY. 


\section{$2 \mathrm{BHL}$ Biodiversity Heritage Library}

Chen, Luetta. 1939. "Notes on Chinese Bauhinia with Description of Three New Species." Journal of the Arnold Arboretum 20(4), 437-439.

https://doi.org/10.5962/p.325791.

View This Item Online: $\underline{\text { https://www.biodiversitylibrary.org/item/33596 }}$

DOI: https://doi.org/10.5962/p.325791

Permalink: https://www.biodiversitylibrary.org/partpdf/325791

\section{Holding Institution}

Missouri Botanical Garden, Peter H. Raven Library

\section{Sponsored by}

Missouri Botanical Garden

\section{Copyright \& Reuse}

Copyright Status: In copyright. Digitized with the permission of the rights holder.

Rights Holder: Arnold Arboretum of Harvard University

License: http://creativecommons.org/licenses/by-nc-sa/3.0/

Rights: https://biodiversitylibrary.org/permissions

This document was created from content at the Biodiversity Heritage Library, the world's largest open access digital library for biodiversity literature and archives. Visit BHL at https://www.biodiversitylibrary.org. 